\title{
Critical Issues in Reprocessing Single-Use Medical Devices for Interventional Cardiology
}

\author{
Francesco Tessarolo ${ }^{1}$, Iole Caola ${ }^{2}$ and Giandomenico Nollo ${ }^{1,3}$ \\ ${ }^{1}$ BIOtech, Interdepartmental Center on Biomedical Technologies, University of Trento, \\ 2Department of Microbiology and Virology, Azienda Provinciale per i Servizi Sanitari, \\ ${ }^{3}$ Department of Physics, University of Trento, \\ Italy
}

\section{Introduction}

\subsection{Single-use vs. multiple use medical devices}

During the 1960s and the early 1970s, most medical devices made of glass, rubber, or metal were generally considered to be reusable. This concept did not change until the late 1970s, when medical devices started to enter the market labelled "single-use only".

During the same decades, clinical medicine has undergone substantial changes, with traditional open surgical procedures giving way to new minimally invasive techniques such as endovascular and laparoscopic intervention. Such procedures required new instruments allowing delicate and complex manipulations through small incisions, with the effector portion of the device located some distance from the operator's hand, demanding stable and predictable performance. During this same period, patients and clinicians have become increasingly concerned about the risk of infectious disease transmission, particularly human immunodeficiency virus and hepatitis $\mathrm{B}$ and $\mathrm{C}$ viruses.

One solution to both demands was found in single-use devices (SUDs), shaped from newly developed fabrication materials, firstly polymers, and intended to be discarded after use on a given patient. Consequently, the past three decades have seen an explosion in the production and use of single-use medical devices, stemming from a desire to improve product performance and minimize the potential for disease transmission, and enabled by advances in manufacturing techniques.

\subsection{SUDs reprocessing}

Although a number of advantages are related to the use of disposable goods in medicine, single-use devices are typically more costly on a per-use basis. SUDs are relatively expensive to purchase and their one-patient/ one-product nature made necessary enlargement of hospital inventories and the resulting stream of medical waste.

These aspects have led to the interest in reprocessing and reuse of these devices. Many hospitals began to explore the reprocessing and a limited reuse of products intended for single use, initially using on-site facilities as they have traditionally done with multiple-use metallic surgical instruments. As single-use products became more complex, hospitals began to turn to third-party reprocessors to handle reprocessing needs. 
Differently from the simple re-sterilization, the reprocessing practice is generally perceived to mean the cleaning, disinfection and sterilization of a medical device, including related procedures, as well as the functional testing and repackaging, carried out on a medical device after it has been put into service (EU Public Consultation, 2007).

Reprocessing and reuse of SUDs have gained wide popularity in the last years as a result of the escalating cost of health care. A survey conducted in 2000 revealed that approximately $20 \%-30 \%$ of hospitals in the United States reprocess SUDs (GAO, 2000). Data are likely underestimated because hospitals tend not to report their use of reprocessed SUDs. According to the United States General Accounting Office, substantial cost savings can be achieved by reprocessing SUDs because the cost of in-house reprocessing can be less than $10 \%$ of the cost of a new device and the cost of third-party reprocessing is approximately $50 \%$ of the cost of a new device (GAO, 2000). A national survey in Canada, investigating the current practices of reprocessing and reusing SUDs in Canadian acute-care hospitals indicates that $28 \%$ of hospitals participating in the survey reprocessed single-use devices (Polisena et al., 2008), and gave an overview on the types of SUDs most frequently reprocessed at acute-care hospitals in Canada.

The basic and legitimate questions before starting a reprocessing policy are: i) Are reprocessed SUDs as good as the original devices in terms of chemical and physical characteristics? ii) Is it safe and economically convenient to use reprocessed SUDs? iii) How should reprocessing be regulated to ensure public health?

In a commentary on reusing SUDs it is agreed that the answer to these questions depend on to whom they are addressed (Quian \& Castaneda, 2002). Most of the opposition against reprocessing of SUDs comes from the original equipment manufacturers (EUCOMED, 2002), whereas the medical community is generally supportive of reprocessing (Lindsay et al., 2001). In general, legislation advocates the precautionary approach till scientific evidences are sufficient to guarantee safeness and efficiency. Anyhow, commentary in the scientific literature on this subject is relatively sparse.

\subsection{The current status about reprocessing SUDs}

Nowadays, the practice of reusing SUDs prevails in almost all developing countries of Africa, Asia, Eastern Europe, Central America, and South America, where there are shortages of medical supplies and financial resources (Quian \& Castaneda, 2002). The rationale behind the reuse of disposable devices in these countries is simple and forceful. The overwhelming public demand for minimally invasive procedures made the single-use of devices financially and ethically unsustainable: otherwise, only those patients with sufficient resources would avail themselves of these new procedures because public institutions could not afford the use of disposable devices for the indigent population (Ruffy, 1995).

The evidence for the safety and effectiveness of reusing SUDs is indirect with the majority of studies set in laboratory contexts evaluating surrogate outcomes such as medical device integrity and contamination after reprocessing. Few studies involved outcomes directly related to patients. It is difficult to define adequately a direct causal link between patient exposures to contaminated or faulty medical devices and adverse patient outcomes due to a lack of data on cross-infection and loss of device functionality.

Conflicting results comes from the available studies. Some studies concluded that the reuse of SUDs is potentially safe and effective with strict reprocessing protocols and standards. 
Others do not recommend reprocessing and reuse because the evaluated devices were not clean or sterile and changes in device integrity were evident. These conflicting results were apparent for anaesthesia devices (Daggan et al., 1999; Lipp et al., 2000), airways devices (Vezina et al., 2001), and disposable plastic trocars (Chan et al., 2000; Roth et al., 2002). The reprocessing and reuse of sphincterotome devices was considered safe and effective with proper reprocessing standards (Kozarek at al., 1999). Studies investigating biopsy forceps consistently showed that reprocessing standards were not met as the devices were not clean nor sterile (Hambric 2001, Kinney et al., 2002). In general, there are ethical constraints in using patients in studies designed to determine the 'risk' associated with reusing SUDs, thereby limiting the overall evidence base. However, despite the existence of some recommendations and protocols governing the reuse of SUDs many items are still being reprocessed and reused without definitive evidences on the safety of these practices. Reports of cases where the use of reprocessed medical devices intended for single-use have caused harm to patients are scarce. It is thus difficult to estimate the incidents frequency as, in general, the personnel involved is likely to be reluctant to report the incidents for insurance or other reasons. In most European countries, the reporting of incidents is not mandatory. In the United States, reporting of incidents involving medical devices is mandatory and all reported incidents are integrated into a searchable database. However, when analysing the reported incidents, the Government Accounting Office did not find any evidence that reprocessed SUDs caused more incidents than other devices (GAO, 2008).

\section{Reusing SUDs in interventional cardiology}

Nowadays, one of the few areas where reprocessing and reuse of SUDs seems suitable both for safeness and cost effectiveness is interventional cardiology (Lindsay et al., 2001; Bourassa, 1996, CETSQ, 1994; Krause et al., 2000; Day, 2004). Radiofrequency catheter ablation and percutaneous transluminal coronary angioplasty have emerged as important therapeutic options for patients suffering respectively from a variety of arrhythmias and coronary diseases. A significant portion of the cost for the procedure is represented by the cost of the multielectrode diagnostic and ablation electrophysiology catheters (EP) or the coronary angioplasty balloon catheter (PTCA). Both catheter types are nowadays labelled and marketed as single use only.

Similarly to other SUDs, in the past, as the demand for disposable equipment rose, hospital administrators and physicians began to notice that some products labelled "single use only" were similar to devices that had been formerly distributed as "reusable". It was reported about a letter by one of the major cardiac catheter manufacturer that stated, "our manufacturing processes of Woven Dacron Intracardiac Electrodes have not changed. These electrodes are made with the same materials and in the same manner they have been in the past" (CCHR, 2000). In response to what many physicians and hospital administrators perceived as an arbitrary labelling policy, the practice of reprocessing SUDs evolved to reduce costs and the amount of medical waste. As this practice encompassed critical devices such as electrophysiology and PTCA catheters the complexity of decontamination and sterilization procedure increased. The role of hospital committees (physicians, nurses, infection control specialists, risk managers, hospital lawyers, and professional reprocessors) evolved to monitor the safety of repeocessing methods. Many hospital administration believed this practice was safe, some made use of third party reprocessors, and others abandoned the practice altogether. 
In the present context, material and technological advancements brought to produce and place on the market high-quality and technologically advanced devices for interventional cardiology with higher therapeutic efficiency but considerably more expensive interventions. Considering the worldwide shortening of economic resources in healthcare systems, the issue of reuse and reprocessing feasibility in a field like interventional cardiology, reveal a great interest and represent a very topical problem.

\subsection{The clinical knowledge on reprocessing SUDs in interventional cardiology}

The issue of reprocessing single use devices in interventional cardiology has been debated from many years and literature presents some investigations which have been conducted to explore technical feasibility, safety, and efficacy of introducing a reprocessing policy in hospital and health care institutions (Bloom et al., 1997; Blomstrom-Lundqvist, 1998; Azyman et al., 2002; Brown et al., 2001; Browne et al., 1997; Chaufour et al., 1999; Granados et al., 2001; Luijt et al., 2001; Ma et al., 2003). Available scientific evidences are of utmost importance for in deep addressing this topic and pointed out the need for new experimental data on technical feasibility, and clinical effectiveness since new materials, manufacturing advancements and substantial technological improvements are frequently introduced in the production of new medical device generations.

Some clinical studies tried to convey clinical data on safety and efficiency by introducing reprocessing and reuse practices in the interventional context and retrospectively or prospectively evaluating patients' outcome. Moreover, some case studies highlighted important benefits and limitations of the reprocessed instrumentation that arose during clinical reuse. All these evidences will constitute the starting point for approaching the reprocessing issue and formulating recommendations and guidelines even more efficient and precise.

The issues pertaining to the safety and efficacy of reusing catheters focus on the risk of transmitting an infection from one patient to an other and the structural and functional integrity of a catheter that is used more than once on different patients. Differently from resterilization procedures that are quite well established, protocols for SUDs disinfection and cleaning are often lacking or improperly designed. Moreover, objective procedure for the measurement of catheter integrity and functionality are not as well documented since they are highly related to materials and design. Some catheters are subjected to very little stress during a procedure, while the deflectability or manoeuvrability of others may change considerably. Lumen cleaning, disinfection and patency are critical due to the peculiarity of catheter design and, sometimes, rapid and effective procedures are to be implemented. Moreover, reprocessing may affect catheter materials and could have a significant impact on functionality.

\subsection{Electrophysiology and ablation (EP) catheters}

A few published studies have evaluated the safety of reusing catheters for electrophysiological studies and have addressed some of these issues. O'Donoghue and Platia surveyed 12 medical centres to determine the safety of reusing EP catheters (O'Donogue \& Platia, 1988). The incidence of infection related to a total of 14640 electrophysiological studies, involving 48075 catheter uses, was reported. At three centres, catheters were automatically discarded after a single use. These centres carried out 1245 electrophysiological studies using 3125 catheters. At the other nine centres, the catheters 
were sterilized for reuse. There were 13395 interventions using 44950 catheters in the reuse group. The incidence of bacteraemia (blood borne infection) and superficial skin infection at the site of catheter insertion were respectively $0.03 \%$ and $0.03 \%$ for the single use group and $0.018 \%$ and $0.002 \%$ for the reuse group. The authors concluded that sterilization and reuse of the catheters used in this study did not result in an increase in the risk of infection. They felt the catheters were sufficiently durable to be reused well in excess of five times, and that one-time use of such catheters appeared to be an unnecessary and expensive policy.

Dunnigan et al. obtained similar results in a prospective study that evaluated catheter reuse over a 5-year period during which 178 catheters were used 1576 times for 847 electrophysiological studies (Dunnigan et al., 1987). No complications were encountered during the study period. All reused catheters were effective for cardiac pacing and recording of cardiac electrical signals. Surveillance cultures and biological indicators revealed that adequate sterilization procedures were used. The authors concluded that electrophysiological catheter may be safely reused provided a thorough cleaning, testing, and record keeping system is instituted. They also concluded that the practice of reusing catheters would result in substantial cost savings to hospitals.

The clinical trials presented above were conducted in patients undergoing diagnostic electrophysiological studies before the advent of deflectable catheters and arrhythmia ablation procedures.

Avital et al. prospectively investigated the time course of electrical, physical, and mechanical changes in ablation catheters to determine the effect of reuse on safety and efficacy (Avital et al., 1993). They studied 69 ablation catheters made by a single manufacturer that were used in 336 procedures. Testing of physical integrity consisted of visual and stereoscopic examination of handle function, catheter shaft, and the deflectable tip. Specific attention was paid to the ablation electrode attachment to the catheter shaft, and the ablation tip electrode was scrutinized for pitting. The electrical integrity of the catheters was checked by measuring the electrical resistance from the handle connector to the recording rings and to the tip electrode. Deflection and torque measurements were made to assess mechanical integrity. During the course of this study, 36 catheters (52\%) were rejected at some point because of mechanical or electrical failure. Eighteen catheters were repeatedly sterilized and 11 of the catheters were used 10 times. The most common reasons for catheter rejection were tip electrode glue separation after $4.3 \pm 4.3$ uses and loss of deflection after 5.0 \pm 3.3 uses. The glue that covers the most proximal portion of the distal electrode was shiny and uniform before any use. The application of radiofrequency energy causes a rise in tissue temperature and the electrode tip is heated secondarily. Small fractions of glue were missing and may have been released into the bloodstream. Catheters with blood that collected in this space could not be properly cleaned. There was no evidence that the tip to shaft attachment was affected by the outer glue separation; however, the possibility that the attachment of the tip electrode was weakened by the glue separation was not excluded by the authors. Electrical discontinuity was observed after $10.0 \pm 3.7$ uses. There was no significant decrease in the catheter torquing ability that determines the steering responsiveness of the catheter. The medical records of 140 patients who had arrhythmia ablation procedures in this study revealed only one case $(0.7 \%)$ of local infection at the insertion site that was treated effectively by antibiotics. There were no other complications.

Avital and co-workers concluded that the catheter model used in this study could be reused an average of five times. They recommended that, after each use, catheters be carefully examined 
under magnification with special attention to the tip electrode. They also recommended that the catheters be tested for deflection and electrical integrity after each use.

As part of an internal quality review process Aton et al. determined the effects of reprocessing on mechanical integrity, sterility, and chemical residuals to establish and validate an institutional policy for reuse (Aton et al., 1994). A total of 12 commercially available catheters from two manufacturers were analysed. Eleven of the catheters were randomly selected from the catheter inventory of the clinical electrophysiological laboratory after being used one to four times. They were manually cleaned, repackaged, and gas sterilized with ethylene oxide. To assess the sterility of reused catheters, three were cut into 2 -inch segments, placed in bacterial culture media, and incubated for 5 days. Six of the catheters were analysed for chemical residuals after gas sterilization. Two catheters were examined for evidence of component failure. Visual inspection and microscopy were used to determine the mechanical integrity of the catheter surface, and x-ray inspection was performed to assess interior structures.

The study results of Aton et al. showed no bacterial growth detected on any of the cultures, which indicated that reprocessed electrode catheters are effectively sterilized. The chemical analysis demonstrated that the concentrations of ethylene oxide detected in extraction liquid exceeded standards established by the FDA. Microscopic examination of reprocessed catheters demonstrated inconsequential metal and fibre particulates on the catheter surface and at some electrode to catheter interfaces. Fluid entrapment around the distal pole may occur in catheters with tip electrodes. The shaft of the catheters and the electrodes remained intact. No evidence of electrical discontinuity was found and the integrity of the internal structures was confirmed by x-ray inspection. The authors concluded that, with the sterilization techniques frequently used at that time by hospitals, the potential for chemical residual contamination might exist after sterilization with ethylene oxide.

\subsection{Percutaneous coronary angioplasty (PTCA) catheters}

Similarly to EP catheters, a few clinical trials were performed to assess safety and efficiency of PTCA reprocessed catheters and only a single randomised, double blind, clinical trial was found in the English literature.

In 1994 a first relevant and debated study by Plante et al. was designed to determine the effectiveness, safety and costs associated with reuse of angioplasty catheters and to compare these results with those of a contemporary centre that employed a single-use strategy in Canada (Plante et al., 1994). In a prospective observational study, data forms were completed after each angioplasty procedure and before patient discharge over a 10-month period. A total of 693 patients were enrolled in the two centres. Clinical and lesion characteristics were similar except for a higher incidence of unstable angina at the reuse centre. The angiographic success rate was identical (88\%) at both centres, but the reuse strategy was associated with a higher rate of adverse events, prolonged procedure time and increased use of contrast medium, especially in lesions that were not crossed by the initial balloon and in patients with unstable angina. Whether these differences are related to the reuse strategy or to differences in patient groups cannot be ascertained by this observational study as pointed out by Rozeman and colleagues (Rozemann et al., 1995).

Mak et al. re-evaluated clinical data from Plante's study using a multivariate statistical model with the purpose to control for the differences in the baseline clinical characteristics of the patients at the two centres and to determine if catheters reuse was associated with an 
increase in complication rate (Mak et al., 1996). The reanalysis showed that the reuse of balloon catheters was not associated with an increased in-hospital event.

A study conducted in the United States by Browne and co-workers aimed at evaluating the performance of angioplasty catheters, restored under a strict remanufacturing process (Browne et al., 1997). Used PTCA balloon catheters were shipped to a central facility and were decontaminated, cleaned and tested for endotoxins. Physical testing and quality assurance were performed before the products were packaged and sterilized with ethylene oxide. Catheter performance was assessed in a pilot study powered to detect a $5 \%$ difference in the angiographic failure rates of new and reused balloons. Under specific indication for PTCA procedure, 107 patients were enrolled, 106 had a successful laboratory outcome, and 1 required coronary artery bypass graft surgery after failed rescue stenting. Over 122 lesions attempted, the angiographic failure rate was $7 \%$ (10 of 108) comparable to the $10 \%$ rate seen with new balloons in other studies. Authors concluded that restoration of disposable coronary angioplasty catheters using a highly controlled process appears to be safe and effective, with success rates similar to those of new products and no detectable loss of performance. Moreover the reported cost analysis suggested that implementation of reuse technology for expensive disposable equipment may offer cost savings for U.S. hospitals, without sacrifice of quality.

Shaw et al. examined the effects of catheter reuse on duration of PTCA procedures and clinical outcomes by retrospectively analysing clinical data of two patients group isolated before and after July 1996 in Canada (Shaw et al., 1999). In July 1996, because of concern regarding the possible transmission of Creutzfeldt-Jakob disease, the province of Quebec stopped the reuse of PTCA catheters. Prior to this time, PTCA balloon catheters were commonly used a maximum of four times in the enrolled health centre. After this time, only new catheters were used. Fifty-three consecutive patients undergoing PTCA prior to 21 July 1996 were compared with 54 consecutive patients undergoing PTCA after that time. It was concluded that there were no significant differences between the single-use and reuse groups with respect to baseline characteristics, no significant differences in the numbers of PTCA catheters used (97 vs. 103) or angiographic success rates (88\% vs. 83\%). Authors stated that, if catheter reuse is not found to be associated with infectious disease transmission, its widespread use should be considered.

The first randomised, double-blind, controlled, single centre, clinical trial has been performed by Zubaid and colleagues (Zubaid et al., 2001). The study compared the safety (clinical success) and efficacy (angiographic success) of reused versus new coronary angioplasty balloon catheters on a total of 377 procedures, 178 performed by reused catheters and 199 by new catheters. No significant differences in clinical or lesion characteristics between the two approaches were found. The incidence of first balloon failure in reused catheter was similar to that of the new catheter (7\% vs. $5 \%)$ and the angiographic success rate was also similar with 176 cases (98.9\%) in reused catheters and 196 cases $(98.5 \%)$ in new catheters. The number of balloon catheters used per lesion, amount of contrast, and procedural and fluoroscopy time were similar in the two arms. At 30 days, the incidence of major adverse cardiac events was similar in both cases (4.5\% vs. $5 \%)$. The study concluded that, in a wide variety of patients, the clinical results of reused balloon catheters are similar to those of new catheters and reused catheters are as effective and safe as new catheters.

A prospective randomised trial comparing new and reprocessed balloons, including stented and stand-alone balloons, has been more recently proposed by Unverdorben and colleagues 
(Unverdorben et al., 2005). Percutaneous coronary interventions were performed randomly in 238 consecutive patients with either new or 1 to 3 times reused balloon catheters. Crossing of the stenosis decreased from $96 \%$ with new balloon catheters to $93.2 \%$, with 1 time reused balloon catheters to $81.8 \%$ with 2 times reused catheters and to $80.8 \%$ with 3 times reused catheters. In all primary failures using resterilized balloon catheters, new ones of the same nominal diameter were successful. The angiographic follow-up rates were 77.4 $\%$ for new balloon catheter, $79.5 \%$ for 1 time reused balloon catheters, $75.0 \%$ for 2 times reused balloon catheters, and $80.8 \%$ for 3 times reused balloon catheters. The percent stenosis was higher in reused versus new balloon catheters, as was the restenosis rate. There was one death in reused balloon catheter category but no event of myocardial infarction. Rates of target lesion revascularizations were similar in stent recipients and more frequent after stand-alone balloon angioplasty with reused versus new balloon catheters. According to clinical data, authors concluded that the use of two or three times resterilized balloon catheters does not seem to be justified in stand-alone balloon angioplasty of de novo coronary stenoses and should be limited to stent procedures until data will be available for other indications.

\subsection{Limitations of available clinical data}

Some criticisms could be made of the above reported studies. Retrospective surveys might depend on the memory of those who responded. Isolated events could escape the attention of the participants in the survey or they may have forgotten complicating events. Some of the prospective studies involved small numbers of patients. In general the methodology of these studies varied and is unlikely to meet recognized standards. Specifically, reprocessing procedures are not harmonized and sometimes not clearly reported. It is also unclear if the protocols used to reprocess the devices were insufficient or if the devices could indeed not be properly reprocessed. Studies claiming safety of reprocessed device do not cover any form of long-term observation of patients regarding the development of infectious diseases and/ or immunological complications following exposure. Moreover, the catheters used in the majority of these studies are old designs. Because changes in materials or/ and functions might have a significant impact on the durability of electrophysiology and angioplasty catheters, it should not be assumed that prior safety data is applicable to new catheter designs that are now adays used in the clinical practice.

This chapter aims at eliciting, discussing and integrating recent experimental findings for the assessment of a reprocessing policy on interventional cardiac catheters labelled as "single use only". The experimental techniques here reviewed supply new parameters for the assessment of quality and safety of reprocessed devices before starting a clinical trial on patients. To this end, technical data and legal, ethical, and economic issues are integrated in order to define the applicability and suitability of SUDs reprocessing.

\section{Technical issues}

\subsection{Chemical and physical analysis of new and reprocessed devices}

The reprocessing protocol should be conceived and designed according to the peculiar characteristics of the device to reuse (Fig. 1). Manufacturers of reusable devices are required to specify in details the proper cleaning, disinfection and (whenever required) sterilization methodologies for guaranteeing a safe and effective reuse on patient. Differently, disposable devices, labelled as "single-use only" do not provide any information addressing for 
reprocessing procedures. Moreover there is a diffused scarcity of materials and designs details on the accompanying documentation. This lack of technical data demands for a complete and exhaustive characterization of the device, posing a particular attention to materials, coatings, and design.

Tessarolo et al. applied a wide number of experimental analytical technique to address essential information about polymer composition and fillers, metals and coatings, surface and bulk parameters, mechanical and thermodynamic materials properties, micro and macro design of PTCA and EP catheters. The preliminary characterization of device, allowed to define detergents, procedures, and sterilization methods according to procedural standards, materials composition, and design. Long and narrow lumens of PTCA catheters demanded for ethylene oxide sterilization, while gas-plasma sterilization was applicable for non irrigated EP catheters (Tessarolo et al., 2004a).

The reprocessing feasibility from a technical point of view has been evaluated on nonirrigated electrophysiology and non-stented coronary angioplasty devices produced by the major worldwide manufacturers. Chemical-physical properties have been assessed on both new and reprocessed devices by using advanced analytical techniques for surface and bulk material characterization such as optical microscopy (OM), electron microscopy (EM), atomic force microscopy (AFM), and infrared spectroscopy (IR).
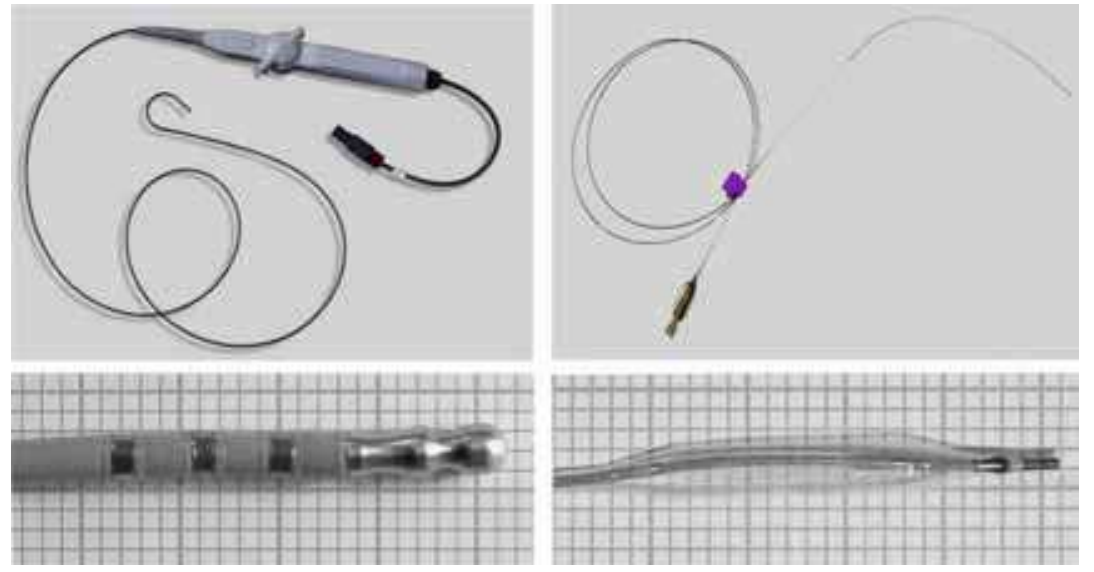

Fig. 1. Representative electrophysiology (upper left) and angioplasty (upper right) catheter for interventional cardiology marketed as single-use only. Images in the bottom show distal portions of the catheters including the functional units: electrodes for recording endocardiac ECG signal and ablation of myocardial tissue (lower left) and an inflated ballon for coronary reprocessing, chemical and physical characterization was carried out at different number of arthery dilation in case of stenosis (lower right). The grid is $1 \mathrm{x} 1 \mathrm{~mm}$ squared. Adapted from Tessarolo et al., 2004a.

To identify device alterations induced by clinical use and/ or reprocessing cycles catheters were characterized after clinical use, simulated reuse, and repeated reprocessing (from 0 to 14 cycles for EP and from 0 to 6 cycles for PTCA) (Fedel et al., 2006; Tessarolo et al., 2004b; Tessarolo et al., 2005; Tessarolo et al., 2006a). OM on EP catheters revealed reprocessingdependent scratches on the polyurethane shaft's surface (Fig. 2) (Tessarolo et al., 2004b). EM and AFM documented a physical-chemical etching on polymers, due to plasma sterilization, 
and a significant increasing in nano-roughness after $7 \pm 4$ cycles of reprocessing (Fig.3) (Tessarolo et al., 2004b).

Residuals of iodate contrast medium in PTCA underlined the need for a timely and efficient cleaning of balloon lumen to avoid crystallization and loss of functionality. Infra-red spectrum suggests that ethylene oxide did not significantly modify polymer's bulk characteristics (Fedel et al., 2006). These studies elicited that materials are highly model dependent and should be verified after each reprocessing cycles. Critical steps for materials modifications were identified in cleaning and sterilization phases.
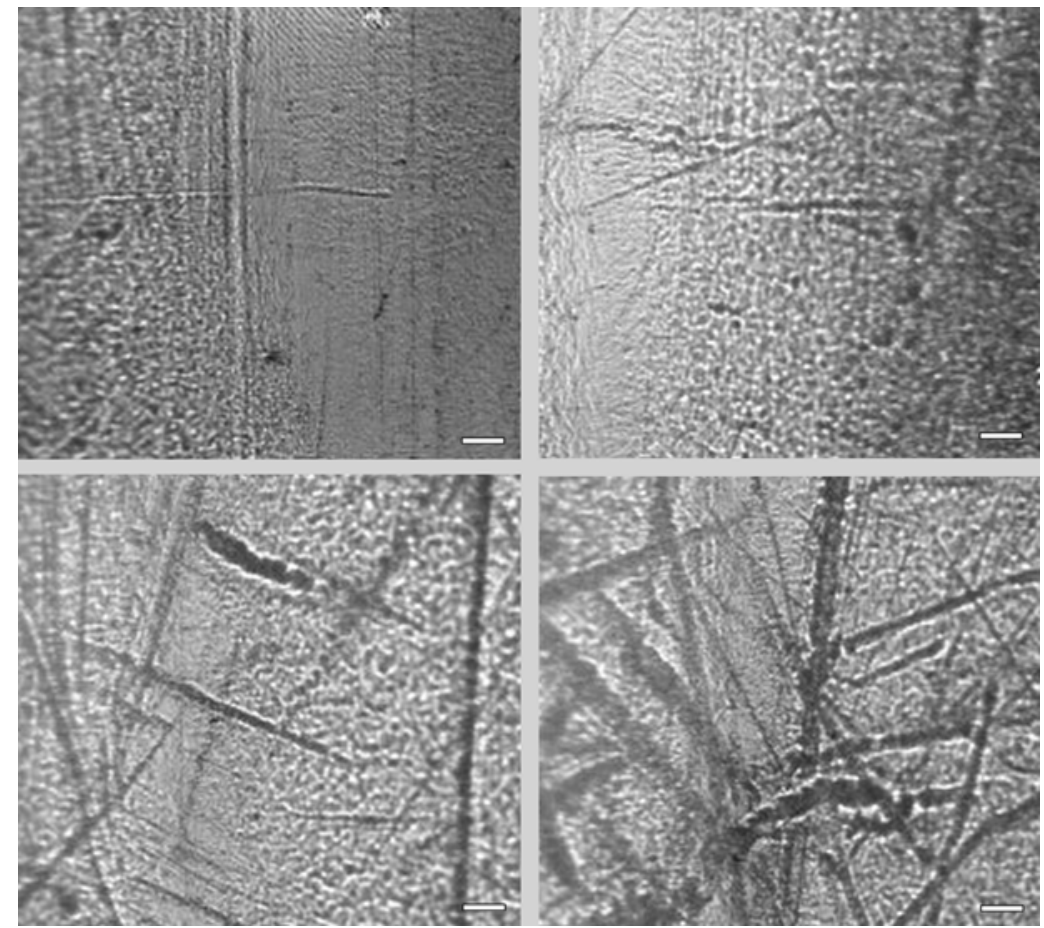

Fig. 2. Optical microscopy on EP catheter shafts. Scratches and indentations were caused by both clinical use and mechanical and/ or manual brushing during cleaning procedures. The amount of scratches was related to the number of reprocessing cycles. From left to right and from top to bottom: new device, 1, 4, 8 times regenerated devices. Bar is $20 \mu \mathrm{m}$.

\subsection{Functional testing of reprocessed devices}

To estimate the maximum number of reuses sustainable by the device in an effective status, functionality was assessed by realizing specific experimental set-ups for both EP and PTCA catheters. Tissue- and organ-synthetic phantoms were realized for simulating clinical use and obtaining quantitative an reproducible functional measurements. Radiofrequency ablation efficiency, electrical characteristics, and catheter slipperiness were quantified in EP devices until 10 cycles of reprocessing (Tessarolo et al., 2004a). Compliance curve, crossing profile, burst pressure, and slipperiness were checked at different steps of the protocol on new and reprocessed PTCA catheters up to three uses (Fedel et al., 2006). 

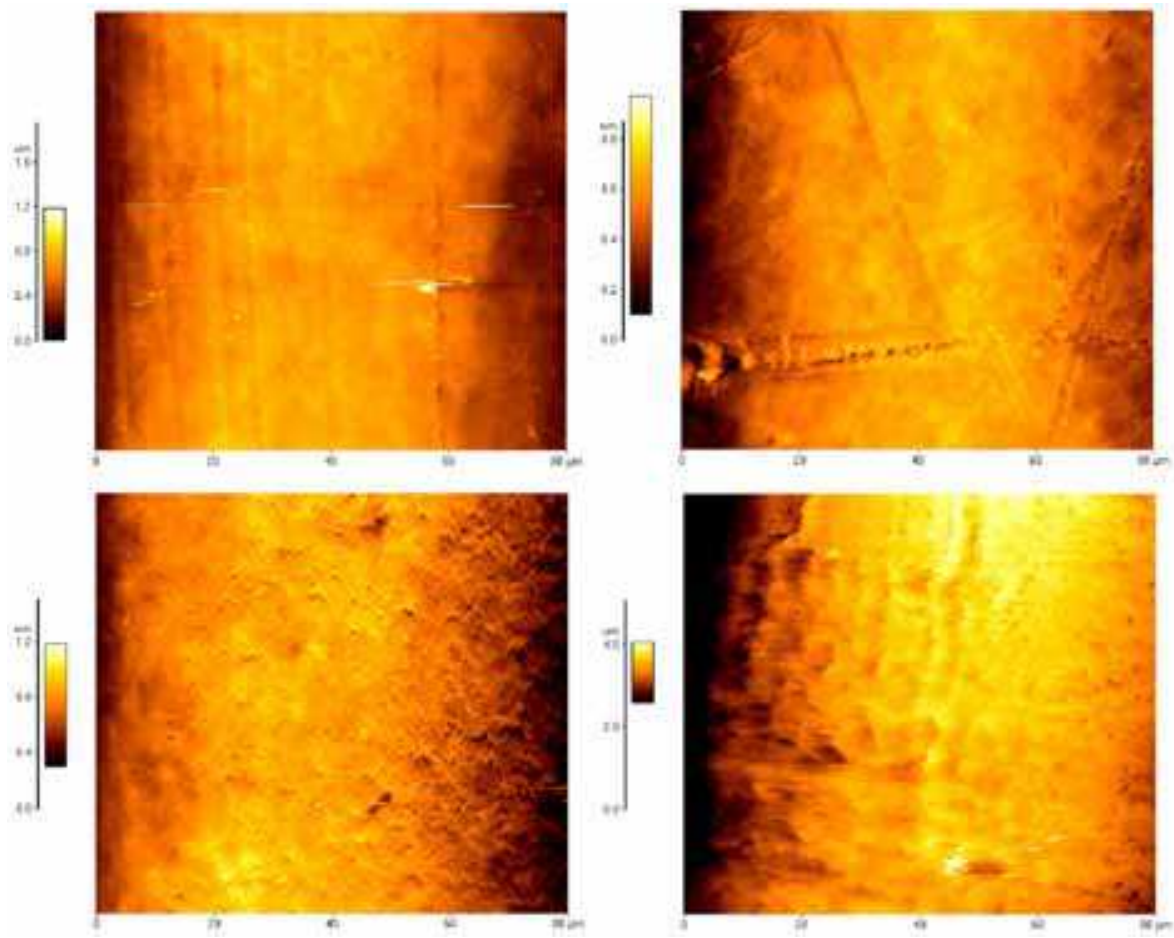

Fig. 3. AFM on EP catheter shaft. Polyurethane underwent progressive nanometric roughening with repetitive gas plasma sterilization. Alterations were induced by the chemical and physical etching of the sterilization technique. From left to right and form top to bottom: new device, 1, 4, 8 cycles regenerated devices. Adapted from Tessarolo et al., $2004 \mathrm{~b}$.

Functionality tests on EP catheters elicited no variations in ablation efficiency, electrodes conductivity, thermometric sensor's precision and accuracy (Tessarolo et al., 2005). Differently, slipperiness tests showed a worsening of lubricious properties in regenerated EP devices after 4 cycles in accordance to the increase of surface roughness. Conversely, functional properties of PTCA catheters were affected by both clinical use and reprocessing procedures (Fig. 4) (Fedel et al., 2006). As a consequence of the mechanical stress in clinical use, balloon diameter at nominal pressure tended to increase. Differently thermo-chemical stress due to cleaning and sterilization induced balloon shrinkage after the first reprocessing cycle. Subsequent cleaning and sterilization did not induce further dimensional alterations. However these modifications did not affect the performance of the device because compliance tests showed the conformity of reprocessed balloons within the $10 \%$ limit of acceptance of manufacturers' original specifications. Anyway, the authors suggested that in case of PTCA catheter reprocessing, it would be profitable to introduce a new calibration curve, with new nominal diameter values. Slipperiness and friction patterns were strictly dependent on PTCA device manufacturer and model but the magnitude of modifications did not compromise in-vitro catheters functionality up to three uses (Fedel et al., 2006). 


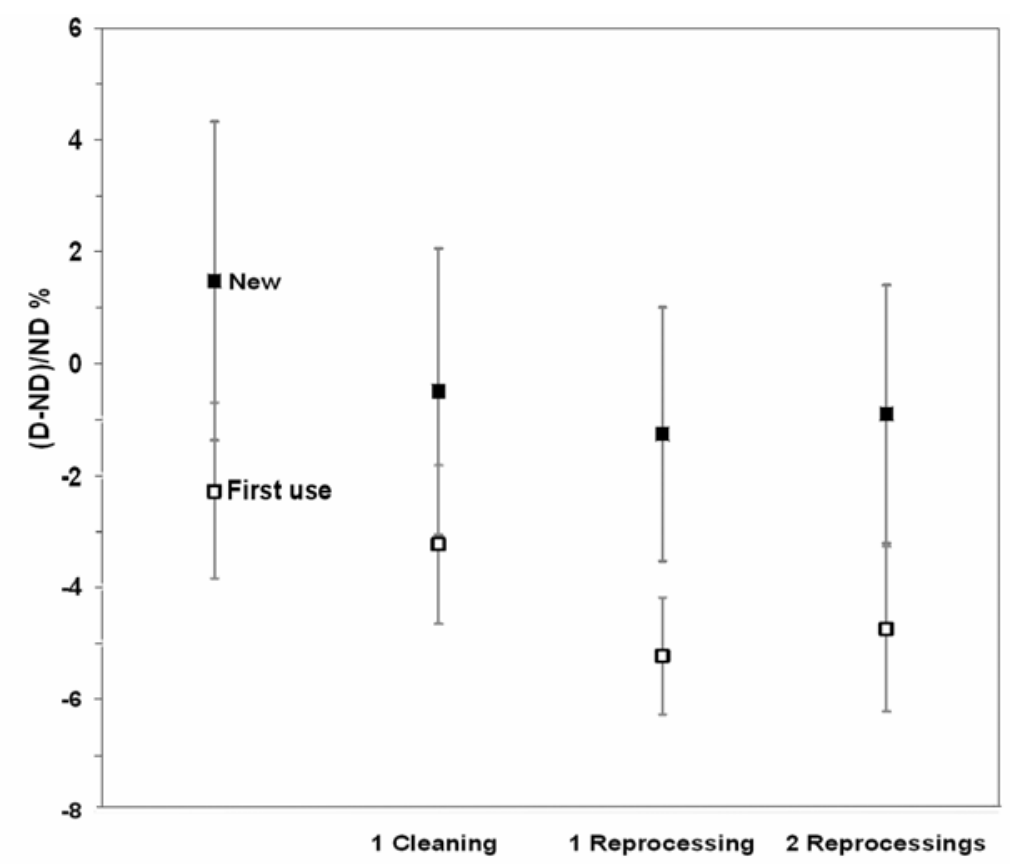

Fig. 4. Effects of cleaning and reprocessing on balloon working diameter (D) normalized to nominal specifications (ND). Data refer to new PTCA devices (full squares), and to products used once on patients (empty squares). The gap between new and used catheters could be caused by exceeding the nominal pressure during in vivo inflation. Both new and used catheters underwent a progressive shrinking after cleaning and first complete reprocessing. Adapted from Fedel et al., 2006.

\section{Hygienic issues}

Hygienic issue should consider a wide spectrum of microbiological tests at different steps of the reprocessing procedure. The bioburden after clinical use and decontamination should be quantified and decontamination-cleaning efficacy, pyrogenic load and device sterility have to be guaranteed. Pathogenic agents/ substances include: bacteria in vegetative or sporulated form, fungi, viruses, microscopic parasites, and prions which are agents responsible for transmissible spongiform encephalopathies. Furthermore, endotoxins (which are part of the bacterial cell wall of Gram-negative bacteria and can be responsible for septic shock) may remain on a SUD even after sterilization as they have a very high resistance to disinfection or sterilization processes. A specific hazard is the possible contamination with agents causing transmissible spongiform encephalopathies as they are particularly resistant to commonly used physical and chemical methods of cleaning, disinfection and/ or sterilization. The causative agent of these diseases consists of the pathogenic isoform of the prion protein, which is misfolded into an infectious agent. It is known that iatrogenic infection of Creutzfeldt-Jakob disease can occur in specific situations associated with medical interventions (Armitage et al. 2009). To date, processes ensuring a total inactivation 
of the transmissible spongiform encephalopathy agents are relatively aggressive precluding their application to materials used for the production of single-use medical devices (Fichet et al., 2004). Anyway, new association of chemical disinfection and low temperature gas plasma sterilization seemed are promising for prion inactivation from thermo-sensitive materials (Rogez-Kreutz et al., 2009).

\subsection{Collection, cleaning and disinfection of used devices}

Tessarolo et al. conducted cultural tests on patient-used catheters to determine and quantify the possible microbial species which could contaminate devices surfaces in clinical procedures (Tessarolo et al., 2004a). Cultural quantitative test on PTCA devices showed that $50 \%$ of the samples were contaminated after use with a microbial bioburden lower than 6 CFU per device (Table 1). Isolated genera were typical of the skin resident microbial flora. Equivalent test on clinically used catheters subjected to decontamination confirmed that inappropriate or untimely procedures might generate bacterial contamination and microbial dissemination in formerly sterile device's surfaces (Table 2). Moreover the use of low quality water might induce contaminations by environmental microrganisms.

\begin{tabular}{|c|c|c|c|}
\hline Catheter & $\begin{array}{l}\text { Bacterial } \\
\text { Load/catheter }\end{array}$ & Isolated species & Notes \\
\hline $\mathrm{A}$ & $6 \mathrm{CFU}$ & $\begin{array}{l}\text { Staphylococcus spp. } \\
\text { Corynebacterium spp. } \\
\text { Aerobial sporigenes }\end{array}$ & - \\
\hline B & $5 \mathrm{CFU}$ & $\begin{array}{l}\text { Staphylococcus spp. } \\
\text { Aerobial sporigenes }\end{array}$ & - \\
\hline $\mathrm{C}$ & $2 \mathrm{CFU}$ & Stophylococcus aureus & - \\
\hline $\mathrm{D}$ & $5 \mathrm{CFU}$ & $\begin{array}{l}\text { Staphylococcus spp. } \\
\text { Corynebacterium spp. }\end{array}$ & - \\
\hline $\mathrm{E}$ & $4 \mathrm{CFU}$ & $\begin{array}{l}\text { Stophylococcus spp. } \\
\text { Corynebacterium spp. }\end{array}$ & $\begin{array}{l}\text { Positive culture of the distal tip } \\
\text { Corynebacterium jeikeium }\end{array}$ \\
\hline $\mathrm{F}$ & $1 \mathrm{UFC}$ & $\begin{array}{l}\text { Staphylococcus. } \\
\text { auricolaris }\end{array}$ & $\begin{array}{l}\text { Positive culture of the lumen } \\
\text { eluate }\end{array}$ \\
\hline $\mathrm{G}$ & sterile & - & - \\
\hline $\mathrm{H}$ & sterile & - & - \\
\hline $\mathrm{I}$ & sterile & - & - \\
\hline $\mathrm{L}$ & sterile & - & - \\
\hline $\mathrm{M}$ & sterile & - & - \\
\hline $\mathrm{N}$ & sterile & - & - \\
\hline $\begin{array}{l}\text { Mean } \\
\text { device }\end{array}$ & $2 \mathrm{CFU}$ & & \\
\hline
\end{tabular}

Table 1. Bioburden on PTCA catheters immediately after use on patients. In $50 \%$ of the examined catheters showed the grow th of typical resident microbial flora of the skin. A very low number of CFU per devices was revealed as outlined in the "mean devices" bacterial load. 


\begin{tabular}{|c|c|c|c|}
\hline Catheter & $\begin{array}{l}\text { Bacterial } \\
\text { Load/catheter }\end{array}$ & Isolated species & Notes \\
\hline $\mathrm{O}$ & $2 \mathrm{CFU}$ & $\begin{array}{ll}\begin{array}{l}\text { Staphylococcus } \\
\text { (CoNS) }\end{array} & \text { spp. } \\
\end{array}$ & \\
\hline $\mathrm{P}$ & $109 \mathrm{CFU}$ & Staphylococcus spp. & $\begin{array}{l}\text { Distal tip: } S . \text { warneri } \\
\text { Lumen eluate: } \text { S. auricolaris }\end{array}$ \\
\hline $\mathrm{Q}$ & $2 \mathrm{CFU}$ & $\begin{array}{l}\text { Staphylococcus warneri } \\
\text { Staphylococcus hominis } \\
\text { hominis }\end{array}$ & - \\
\hline $\mathrm{R}$ & $11 \mathrm{CFU}$ & $\begin{array}{l}\text { Staphylococcus warneri } \\
\text { Staphylococcus spp. } \\
\text { (CoNS) }\end{array}$ & - \\
\hline $\mathrm{S}$ & sterile & - & - \\
\hline $\begin{array}{l}\text { Mean } \\
\text { device }\end{array}$ & $25 \mathrm{CFU}$ & & \\
\hline
\end{tabular}

Table 2. Bioburden on PTCA catheters used on patients and decontaminated. A significantly higher number of CFU per device was revealed in respect to used but untreated devices (See Table 1). CoNS: Coagulase negative staphylococci
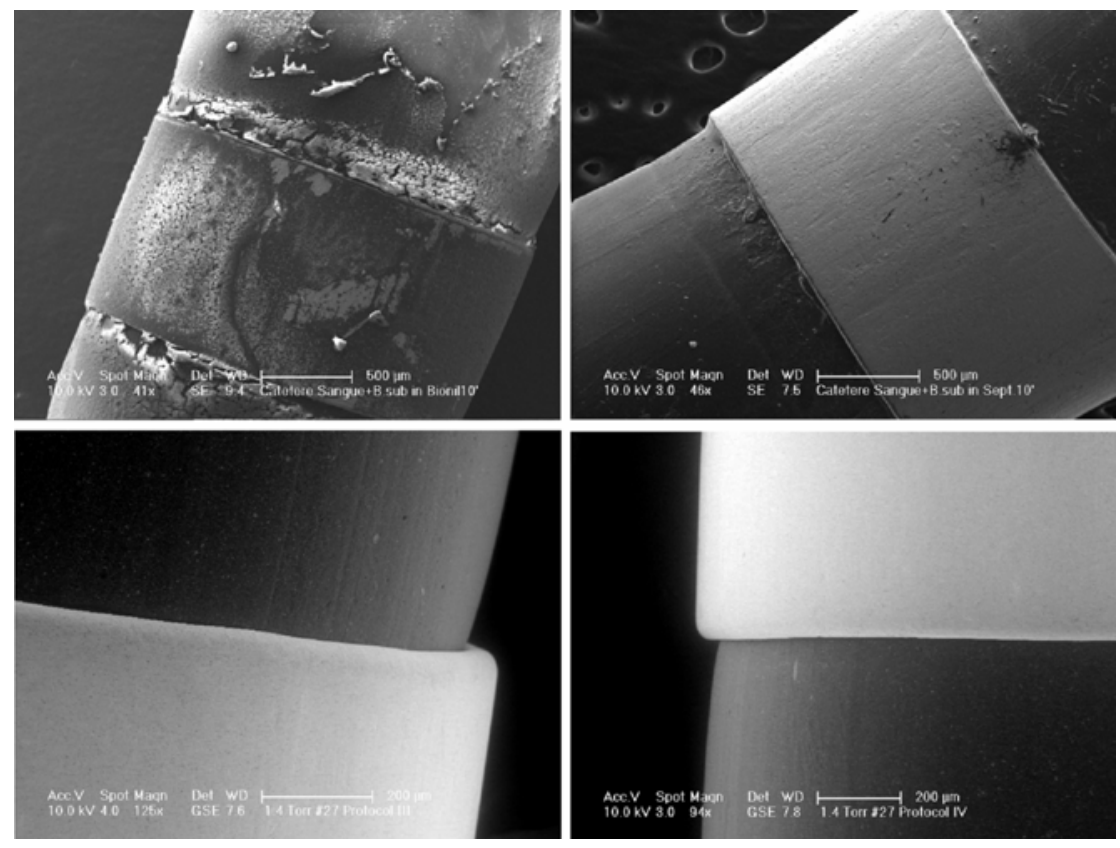

Fig. 5. Scanning Electron Microscopy on decontaminated and cleaned EP catheter by four different protocol: 1) chlorine-enzymatic solutions 2) enzymatic-chlorine solutions; 3 ) polyphenolic emulsion 4) polyphenolic plus enzymatic treatment. From top to bottom and from left to right is reported the electrode-shaft interface of catheter after protocol 1, 2, 3, and 4. Adapted from Tessarolo et al., 2004c and Tessarolo et al., 2007a. 


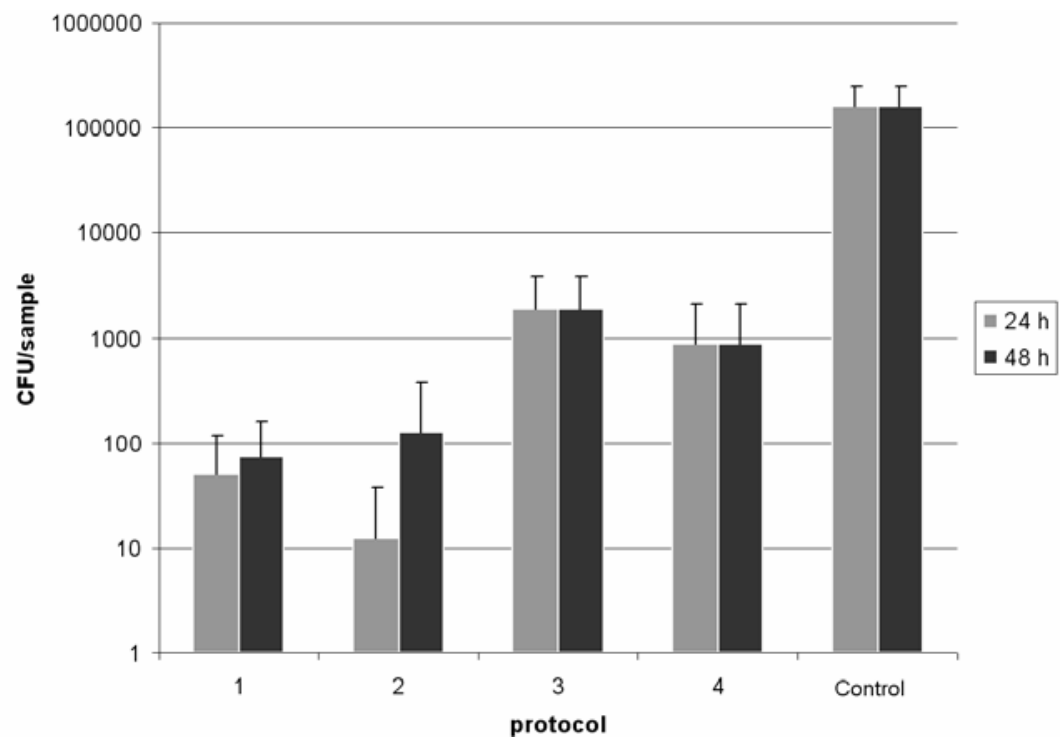

Fig. 6. Survival of $P$. aeruginosa after the exposure of the contaminated catheter shaft to the same four different protocol for decontamination and cleaning described in Fig 5. Colony count was performed at 24 and 48 hours to evidence any eventual bacteriostatic effect. Initial bacterial load (conrol) was $1.6 \times 10^{5} \mathrm{CFU}$ per catheter.
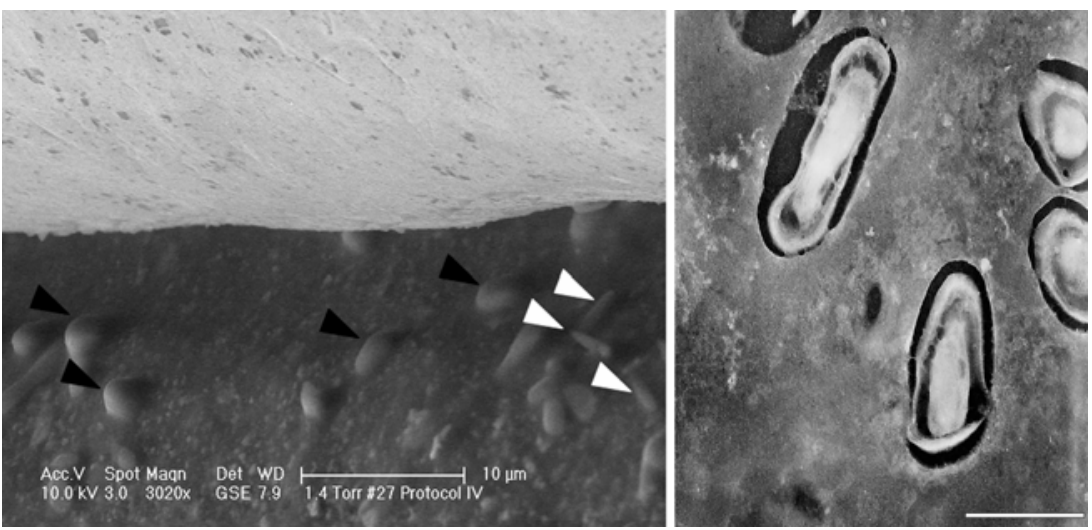

Fig. 7. Electron microscoscopy images of biologic residuals including Bacillus subtilis in catheters processed for resterilization. Left: Low-vacuum SEM at electrode-polymer interface showing bacterial shaped corpuscles embedded in the organic coating residual. Sporulated (black arrowheads) and vegetative (white arrowheads) forms of $B$. subtilis might be associated to this debris according to morphology and size. Right: TEM on a ultrathin section (bar is $1 \mu \mathrm{m}$ ) of blood clot scraped from the catheter surface after treatment by polyphenolic solution and enzymatic detergent. The inclusion of $B$. subtilis in vegetative and sporulated forms are shown. TEM image was negative filtered. Adapted from Tessarolo et al. 2007a. 
Since the efficacy of pre-sterilization device treatments is fundamental for sterilization success, different decontamination, disinfection and cleaning protocols were tested to identify biocide properties and cleaning effectiveness. Tessarolo and co-workers reported about 80 catheters samples, contaminated with bacteria-spiked human blood and subjected to different pre-sterilization protocols including chlorine releasing agent, polyphenolic emulsion, and enzymatic detergent (Tessarolo et al., 2004c; Tessarolo et al., 2007a). Treated samples were analysed by electron microscopy for biologic and inorganic residuals characterization, while cultural quantitative methods assessed chemicals' bactericidal effectiveness. Significant differences by using different chemicals were found. The use of chlorine solution as first treatment left relevant blood residuals on the exposed device surfaces while protocols including the polyphenolic emulsion, realized a deep cleaning of the surfaces with a very limited lasting bioburden (Fig. 5). Interaction and absorption of polyphenols on polymers has to be also considered for potential toxicity in re-use. Cultural quantitative methods showed the highest biocide properties of hypochlorous-acid based protocols while a lower bactericidal activity was documented for polyphenolic based solutions (Fig 6). Authors elicited the need to optimize both the disinfection efficiency and the biologic burden removal. It is also mandatory to provide for protecting the personnel from infectious agents. This threefold aim ask for defining structured protocols based on the synergic integration of mechanical and chemical agents.

Finally, the problem of pyrogenic risk related to reuse of single use devices, got in contact with blood, was specifically addressed (Tessarolo et al., 2006b). With this purpose the pyrogenic status of 61 catheters was monitored in three fundamental steps of the reprocessing protocol: untreated, after decontamination-cleaning procedure and after complete reprocessing. Endotoxin content was assayed by LAL test both after standard clinical use conditions and worst-case contamination by in-vitro high inocula endotoxins spiking. Experimental results demonstrated that standard clinical use did not represent a critical source of endotoxins contamination. Differently, the use of tap water and manual cleaning processing increased the pyrogenic load by introducing gram-negative microorganisms and by favouring bacterial growth on residual moisture. Microbiologically high quality water for limiting gram-negative contamination and overgrowth, is mandatory to avoid pyrogenic risk in reusing single use devices. Microbiological data suggested that the use of automated cleaning system instead of or in addition to manual device processing is more suitable for guaranteeing a reliable and standardized cleaning of complicated designs and sensitive materials.

\subsection{Sterilization of processed SUDs}

High-sensitive and reproducible sterility testing methodologies were developed by Tessarolo and co-workers to evaluate performances and limitations of a regeneration protocol for EP catheters (Tessarolo et al., 2006c). Devices were collected after clinical use on patient, underwent repeated cycles of simulated-use (bacteria spiked blood) and regeneration (decontamination, cleaning and sterilization), and were cultured for 28 days in trypticase soy broth. Sterility tests provided experimental evidences on 208 samples, six cycles of regeneration, and four inoculating bacteria species. Sterility investigations showed no positive sample to the inoculated strain until the fourth cycle of reprocessing (Table 3). The inoculated Bacillus subtilis strain was recovered in samples reprocessed five and six times. These results were in accordance with surface analysis which pointed out alterations on materials' properties that might favour bacterial persistence and limit reprocessing 
effectiveness after repeated reprocessing cycles. Hence, over-reuse of the devices could affect both safeness and efficacy as documented by sterility data and surface worsening after five reuses (Tessarolo et al., 2004b, Tessarolo et al., 2006c). Coming from experimental conditions conducted in worst case scenarios, this estimation of the maximum number of reprocessing cycles was precautionary.

\begin{tabular}{cccc}
\hline Lot & $\begin{array}{c}\text { Tested } \\
\text { devices }\end{array}$ & $\begin{array}{c}\text { Positive devices } \\
\text { to inoculated strain }\end{array}$ & $\begin{array}{c}\text { Positive devices } \\
\text { to inoculated strain \% }\end{array}$ \\
\hline I regeneration & 54 & N.A. & N.A. \\
\hline II regeneration & 36 & 0 & $0 \%$ \\
\hline III regeneration & 24 & 0 & $0 \%$ \\
\hline IV regeneration & 28 & 0 & $0 \%$ \\
\hline V regeneration & 35 & 1 & $2.9 \%$ \\
\hline VI regeneration & 22 & 1 & $4.5 \%$ \\
\hline
\end{tabular}

Table 3. Sterility tests on EP catheters. Regeneration procedures were ineffective in restoring sterility of devices reused more than five times. Data are reported for 2 nd to 6 th regeneration after simulated in-vitro contamination by using bacterial spiked human blood ( $10^{7} \mathrm{CFU} / \mathrm{mL}$.). Due to first patient clinical use, data on possible contaminating species in I regeneration lot are not available (N.A.). Adapted from Tessarolo et al., 2006c.

\section{The ethical and legal context}

\subsection{Juridical issues about reprocessing SUDs}

There is no uniform policy governing the reuse of SUDs in the European Community. Finland, France, Germany, UK, Portugal, Spain and Sweden have all introduced various degrees of regulation (including a total ban) on refurbishing and reuse of SUDs. Despite this, the practice remains present in EU countries.

Directive 93/42/ EEC on medical devices (MDD), adopted on 14 June 1993, stated that medical devices intended for single-use must bear on their label an indication that the device is for single-use. Directive 2007/ 47/ EC, adopted on 5 September 2007, amending Directive 93/ 42/ EEC, provided further clarification defining a "single-use" medical device as "a device intended to be used once only for a single patient". The Directive also introduced the requirement that if the device is for single-use, information on characteristics and technical factors known to the manufacturer that could pose a risk if the device were to be re-used must be provided in the instructions for use. According to the Directive and to national legislations of European countries, producers of medical devices are held to guarantee the number of times the product can be reused, assuming the complete liability during the whole life cycle. A disposable device ends its intended life after the first use so losing any manufacturer's responsibility for subsequent reuse. On the other hand, in most of European countries, no bans are clearly provided by the law for a reprocessor who intends to enter in the market proving a safe reuse of this kind of devices. The freedom of enterprise and the free competition, submitted to strict market regulation, could in fact promote competition and products improvement. Consequently, many European countries assumed that the certificate of conformity system should be extended to the reprocessor's activity, since CE mark is a guarantee for product compliance with all of the essential requirements for medical devices. 
In the United Kingdom, France, Spain, and Switzerland, recommendations, legislation, or notes have been published forbidding or warning on the reuse of SUDs. Conversely, in Germany, the Medical Device Act does not ban the reprocessing of medical devices labelled for single use and advises users and institutions to use their own discretion. Therefore, catheters are processed for reuse in many hospitals in Germany. The regulative answer provided by the German legal system to reprocessing represents a possible balance between the need to maximize the efficiency of the health care system and the safeguard of patient health and safety. German legislation on matter of reprocessing comes from specific definitions in the MDD European directive transposition. In the German case, manufacturer's indication for "single usage" is not considerable in the notion of "intended purpose". This eliminates any implicit ban of reprocessing practice and avoids the assimilation of reprocessor to manufacturer, so considering the reprocessing activity differently from "fully refurbishing". Moreover reprocessing does not entail a placing of the device in the market since after process it is still delivered to the first purchaser who represents the effective owner. This fact allowed to not re-marking the devices with a new CE label. The third party reprocessor provides the possibility of unique identification and the re-delivering to the sole owner. However, according to German regulation, the reprocessor is not exempted from carrying on complex procedures for process control and validation.

The United States Food and Drug Administration increased its oversight of SUDs reprocessing gradually. On August 14, 2000, a new FDA policy entitled, "Enforcement Priorities for Single-Use Devices Reprocessed by Third Parties and Hospitals," was released to regulate third-party and hospital reprocessors of SUDs. Under the new guidelines, these reprocessors are considered device manufacturers. Therefore, third-party firms and reprocessing hospitals have to obtain pre-market approval (PMA) from the FDA for their products and are obligated to follow the same adverse-event reporting requirements (Medical Device Reporting) as OEMs.

The reprocessors, whether third-party firms or hospitals, are also required to register their establishment with the FDA, provide a list of devices they reprocess, establish a medicaldevice tracking system, conform to good manufacturing practice requirements, and follow general labelling requirements regarding the name and site of reprocessing and inclusion of adequate directions for use.

The Australian Government does not endorse the reuse of SUDs and requires informed consent from patients if a reprocessed device is to be used.

Reuse of SUDs was common practice in Canada before august 1996. At that time the government advised to discontinue the practice of reusing SUDs primarily because of concern about the potential risk of blood borne Creutzfeldt-Jakob disease. However, in Canada, there are no Federal or Provincial regulations governing the reuse of single-use medical devices. Currently, Health Canada does not regulate the reuse of medical devices by health care facilities or reprocessing of these devices by third-party reprocessors. The use or reuse of medical devices falls outside the governance of the Food and Drugs Act and the Medical Devices regulations. These acts have authority over the manufacture and sale of medical devices and were never intended as regulations over the use (including reuse) of such medical devices.

\subsection{The ethical issue}

From an ethical standpoint, two main aspects have to be considered: patients safety and distributive justice in allocating available resources. The focus of the concern should be 
upon the ethical obligation of all health care professionals/ institutions to cause no harm or injury to their patients, but the issue is complicated by important considerations involving the appropriate allocation of increasingly scarce health resources. In an era of enormous restriction of resources in the health care system, the incentive to save money is a legitimate claim. From an ethical perspective, any wastefulness in unjustifiable in a health care system where a patient may be denied a service because a lack of resources, (CETSQ, 1994). As such, reuse may not be unethical so long as it is established that the quality of care is maintained and there is no significant loss of device effectiveness and no unreasonable increased risk of harm to the patient. Anyway, economic saving should not be at the expense of patient safety and the focus of any consideration of the practice of reuse must be the patient (NHMRC, 1997).

At the same time it is included in the ethical debate the importance to spread goods and technologies in less privileged countries. It was reported that in different health systems the risk/ efficacy ratio could be substantially different and the most of the clinical work can be done with less technological support than that typically available in more affluent countries (Ruffy, 1995). On a secondary level, hospitals which reuse SUDs may be fulfilling their societal obligations to protect the environment through decreased landfill disposal, providing that the substituted cleaning and sterilization procedures are not of increased harm to the environment (CHA, 1996).

\subsection{Patient's informed consent}

Patients have the right to know and physician should not be reluctant to disclose information about reuse and reprocessing of single use devices to the patient. Both individual patients and public trust requires that openness is exercised and that the practice of reuse is not concealed in any way. A hospital's policy in this regard must therefore be public knowledge and clearly disclosed (CETSQ, 1994). However there are different opinions regarding the need for obtaining patient's consent about reusing SUDs. Usual ethical perspectives on informed consent could be grouped in two different positions.

The first concludes that patients should be always advised when reusing SUDs because the risk of this practice has not been adequately studied. Some ethicists believe, moreover, that the informed consent of a patient is ethically necessary, since there is an obligation on medical staff not to lie, deceive or otherwise interfere with a patient's free choice (Hall, 1991). This opinion is, in some points, also reflected in the original equipment manufacturers (OEMs) position about SUDs reuse. Producer remarks that it is a basic principle of medical treatment that the patient should consciously agree to the form of treatment. It is OEMs' opinion that patient should be clearly told of all relevant factors, including the fact that he is to be treated with a reused single-use device contrary to the manufacturer's instructions, and that this may expose the patient to possible additional risks (EUCOMED, 2002).

The second ethicists' perspective concluded that the need to obtain informed consent for reused SUDs depends on if the physician believes there is an appreciable and significant risk for the patient. In this approach it is supposed that no substantial differences in safety and efficiency are imputable to reprocessed devices in respect to new ones. This perspective considers that the risk of a life-threatening or fatal complication during the clinical intervention is always present. As an example, in the case of electrophysiological studies, such a risk is in the range of 1:1000 (Horowitz, 1986). Conversely, the risk of reusing electrophysiological catheters appears to be so low that no reasonable estimate has been 
identified yet. Relative to the overall risk of the procedure, the risk of reusing the devices might become insignificant.

It is in the opinion of the North America Society of Pacing and Electrophysiology (Lindsay et al., 2001) that, if the use of reprocessed EP devices is not associated with material and functional risk, then there is no ethical reason why this issue must be added to the long list of risks known to be associated with the procedure. Patients should be informed if they ask about the hospital's policy and they have the right to request that reprocessed catheter not be used. The decision to include this discussion when informed consent is obtained should be determined by the attending physician. If a patients objects to the use $o$ a reused catheters it is up to the hospital to decide whether a new catheter will be provided or whether the patient will have to assume the risk of a delay in treatment until a new catheter became available in the course of routine (CETSQ, 1994).

A study on the patient acceptance of reused angioplasty equipment showed that a sufficient number (68\%) of patients would be willing to permit reused PTCA devices (Vaitkus \& Burlington, 1997). The same study pointed out that the disapproval by one third of patient raises the possibility of adverse publicity and litigation for institution implementing a reuse policy. However the perception of duplicity in medical care when informed consent is obtained is of particular concern.

\section{Economic issues}

\subsection{Cost-minimization model}

To estimate the potential saving for budgets of cardiology departments, a cost-minimization model was developed by Capri and colleagues (Capri et al. 2005) and applied to data pertaining to the Italian health system (Tessarolo et al., 2007b; Tessarolo et al., 2009). The model was developed in the hypothesis that reprocessing and reuse of SUDs is performed by guaranteeing safety and efficiency of the reconditioned device as high as the new one.

The model was used to describe the costs associated to catheters for interventional cardiology at departmental level in two different scenarios: single-use policy and re-use policy. Device reprocessing in case of reuse policy was designed by considering a third party professional reprocessor. Accordingly to the model, the single-use catheter's cost $\left(c_{K}\right)$ was computed by the following expression:

$$
\mathrm{c}_{\mathrm{K}}=\mathrm{P}_{\mathrm{K}}+\mathrm{S}+\frac{\mathrm{G}_{\mathrm{K}}}{3 \mathrm{~N}}
$$

Where $P_{k}$ is the new catheter price, $S$ is the cost related to special waste disposal per single device, $\mathrm{N}$ is the total number of used catheters per year in the modelled cardiology department, and $G_{K}$ is the cost for a competitive triennial contracts allocation of new devices. Differently, in case of reprocessing and reuse of cardiac catheters, the expression was modified as follows:

$$
c_{R}=i \frac{P_{K}+(n-1) P_{R}}{n}+(1-i) P_{K}+\frac{S}{n}+C+\frac{G_{K}}{3 N}+\frac{G_{R}}{3 N}
$$


Where $c_{R}$ is the cost for $n$-times used device, $i$ is the reprocessing rate, $P_{R}$ is the reprocessing cost per catheter, $\mathrm{n}$ is the maximal number of uses sustainable by the catheter. Additional parameters were considered, as costs related to collection and handling of used catheter after each use (C), and costs for competitive triennial contracts allocation of reprocessing service $\left(G_{R}\right)$. Potential saving, related to the introduction of a reprocessing SUDs policy, were eventually calculated by the following expression:

$$
\text { Saving } \%=\frac{c_{K}-c_{R}}{c_{K}} \cdot 100
$$

\subsection{Potential saving from SUDs reprocessing in interventional cardiology}

Accordingly to previous finding on safety and effectiveness, the maximum number of uses (n) to enter in the cost-minimization model was set at 6 and 3 for EP and PTCA catheters respectively.

For a cardiology department with a median number of intervention (600 angioplasties and 200 electrophysiological studies per year) the model forecasted a potential saving of about $12 \%$ in the expenditure for PTCA catheter if reprocessing and reuse policy is adopted (Tessarolo et al., 2009). A markedly higher saving of about $41 \%$ and $33 \%$ was computed for EP diagnostic and ablation procedures respectively. The sensitivity analysis on the three main variables, those are regeneration rate, number of uses, and catheter consumption per year, showed that significant differences in savings between EP and PTCA catheters reprocessing are mostly related to the annual catheter consumption that is proportional to cardiac department activity (Fig. 8). Major variations in savings occurred in the range between 1 and 200 catheters per year.

Percent savings generally grew as a function of regeneration rate (i) and maximum number of uses (n), but for high number of catheter usage per year (i.e. greater than 300) there was a tendency to a linear relation between percent savings and regeneration rate, while a plateau in percent saving was reached by increasing the maximum number of uses.

The economic analysis indicated that reuse of SUDs might be a source of savings for the cardiology department. However, the scaling to a specific working unit should be done cautiously. Since the cost saving depends on the number of devices used per year, regeneration might be economically unfavourable if a small number of clinical interventions is performed. The number of catheter used per year is therefore the most immediate parameter for establishing the cut off between benefits and charges in reprocessing SUDs. An additional critical point is the price of new device. Namely, decrease in the cost of new devices could sensibly modify potential saving and, in case of limited percents of benefits as PTCA catheters, a decrease in new device price could nullify the benefit of reprocessing (Capri et al., 2005). Moreover innovations in devices or reprocessing technology could affect the final savings by altering the maximum number of regenerations and the regeneration rate. Anyway, market dynamics forces to make stable the ratio between new catheter prices and regenerated device's cost, usually placed in the range of 0.4-0.5 by third party reprocessor.

Finally, quotes for patient's insurance and risk management should be introduced in the model, and more complex cost-effective analyses and decisional processes have to be applied in case reprocessed device is not as safe and effective as the new one (Sloan, 2007). 

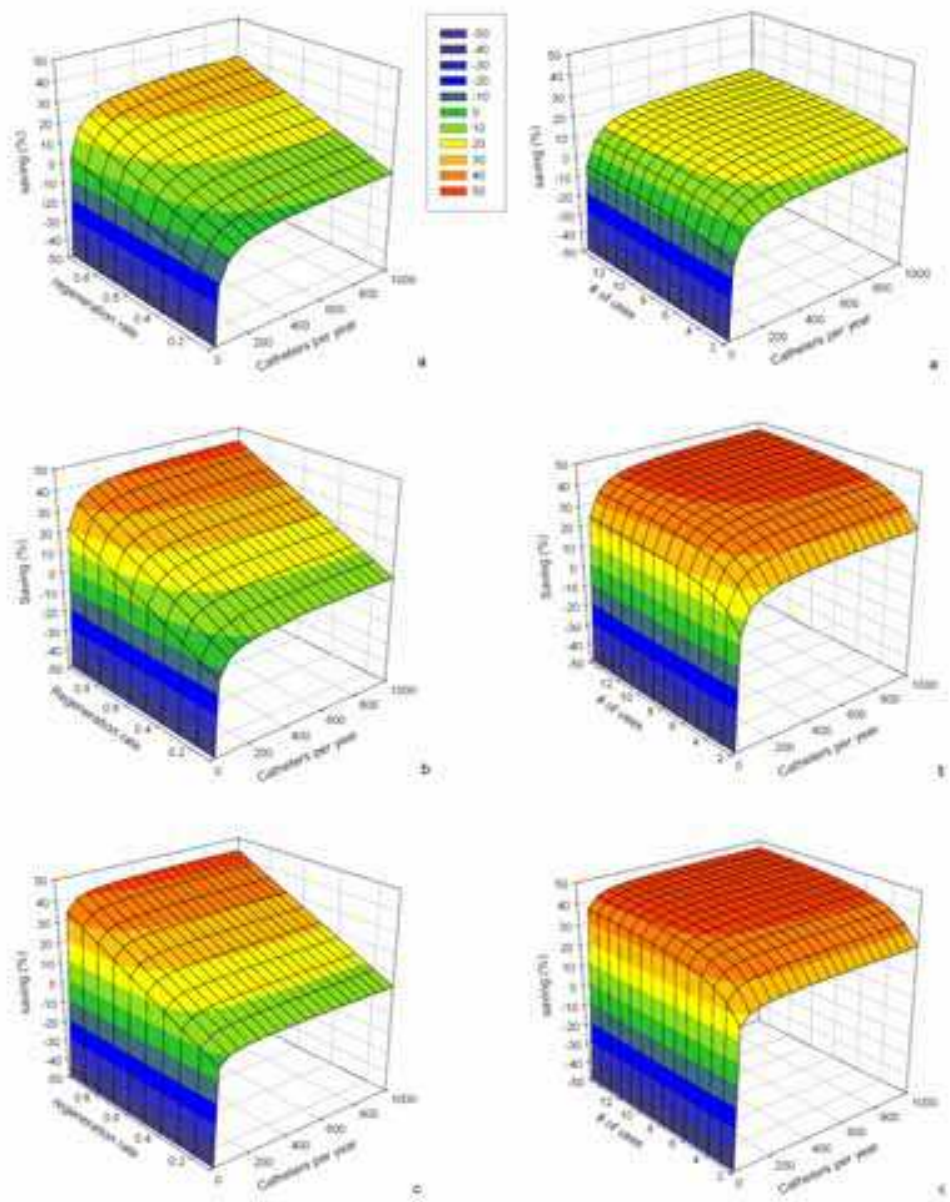

Fig. 8. Sensitivity analysis surface plots for potential percents saving of a reprocessing policy calculated according to the economic model. Left column: variation of the regeneration rate. The number of uses per catheter type has been set to 3,6 , and 6 for (a) PTCA, (b) EP diagnostic, and (c) EP ablation catheters respectively. Right column: variation of the number of uses. The regeneration rate per catheter type has been set to $0.48,0.95$, and 0.95 for (a) PTCA, (b) EP diagnostic, and (c) EP ablation catheters respectively. Adapted from Tessarolo et al., 2009.

\section{Conclusions}

From a technical and hygienic perspective the most efficient and safe reprocessing protocol should contemplate a unique and continuative solution, which provide for all the treatments starting from collection of used devices in cardiology departments to sterilization. This approach to regeneration, while assuring the best hygienic performances, requires devoted infrastructures, trained staff and specific knowledge. These technical considerations added 
to organizational, economic, and legal requirements connected to the need to qualify and certify all reprocessing procedures, suggest the introduction of this practice only in hospitals and health care structures with a significant workload. Anyway the more and more stringent criteria required by legislation and regulative policies underline the need for guaranteeing a certified reprocessing procedure, with the same quality issues supplied by the original manufacturers. These requirements may be unlikely achieved by small or medium hospitals, but could be affordable by relevant health care institutions or by third party industry reprocessors.

\section{References}

Armitage, WJ; Tullo, AB. \& Ironside JW. (2009). Risk of Creutzfeldt-Jakob disease transmission by ocular surgery and tissue transplantation. Eye, 23, 1926-1930.

Aton, EA.; Murray, P.; Fraser, V.; Conaway, L. \& Cain ME. (1994). Safety of reusing cardiac electrophysiology catheters. American eburnal of Cardiology, 74, 1173-1175.

Avitall, B.; Khan, M.; Krum, D.; Jazayeri, M. \& Hare, J. (1993). Repeated use of ablation catheters: a prospective study. eburnal of the American College of Cardiology, 22, 367372.

Ayzman, I.; Dibs, SR.; Goldberger, J.; Passman, R. \& Kadish, A. (2002). In vitro performance characteristics of reused ablation catheters. eburnal of Interventional Cardiac Electrophysiology, 7, 53-59.

Bloom, DF.; Cornhill, JF.; Malchesky, PS.; Richardson, DM.; Bolsen, KA.; Haire, DM. et al. (1997). Technical and economic feasibility of reusing disposable perfusion cannulas. eburnal of Thoracic \& Cardiovascular Surgery, 114, 448-460.

Blomstrom-Lundqvist, C. (1998). The safety of reusing ablation catheters with temperature control and the need for a validation protocol and guidelines for reprocessing. Pacing \& Clinical Electrophysiology, 21, 2563-2570.

Bourassa, MG. (1996). The reuse of single use balloon angioplasty catheters:it is now legitimate? The American eburnal of Cardiology, 78, 673-674.

Brown, SA.; Merritt, K. Woods, TO. \& Hitchins, VM. (2001). The effects of use and simulated reuse on percutaneous transluminal coronary angioplasty balloons and catheters. Biomedical Instrumentation and Technology, 35, 312-322.

Browne, KF.; Maldonado, R.; Telatnik, M.; Vlietstra, RE. \& Brenner AS. (1997). Initial experience with reuse of coronary angioplasty catheters in the United States. eburnal of the American College of Cardiology, 30, 1735-1740.

Capri, S.; Ferrari, P.; Tessarolo, F.; Guarrera, GM.; Fontana, F. \& G Nollo. (2005). Modello di calcolo della convenienza economica per l'ospedale: il caso dei cateteri cardiaci rigenerabili vs. i cateteri monouso. Politiche Sanitarie, 6, 188-196.

CCHR. (2000). Reuse of single use devices. Hearing before the Subcommittee on Oversight and Investigations of the Committee on Commerce House of Representative. Serial $\mathrm{n}^{\circ}$ 106-89. Attacchmnent C, 138. February 10, 2000. US Government Printing Office Washington, 2000.

CETSQ. (1994). Conseil d'Evaluation des Technologies de la Santé du Quebec. The reuse of single use cardiac catheters: safety, economical, ethical and legal issues. Canadian eburnal of Cardioloy, 10, 413-421.

CHA. (1996). Canadian Hospital Association. The reuse of single-use medical devices. Available at: http:/ / sprojects.mmi.mcgill.ca/ heart/ carecha2ca.html. 
Chan, AC.; Ip, M.; Koehler, A.; Crisp, B.; Tam, dS. \& Chung, SC. (2000). Is it safe to reuse disposable laparoscopic trocars? An in vitro testing. Surgical Endoscopy, 14, 10421044.

Chaufour, X.; Deva, AK.; Vickery, K.; Zou, J.; Kumaradeva, P.; White, GH. \& Cossart, YE. (1999). Evaluation of disinfection and sterilization of reusable angioscopes with the duck hepatitis B model. eburnal of Vascular Surgery, 30, 277-282.

Daggan, R.; Zefeiridis, A.; Steinberg, D.; Larijani, G.; Gratz, I. \& Goldberg, ME. (1999). Highquality filtration allows reuse of anesthesia breathing circuits resulting in cost savings and reduced medical waste. eburnal of Clinical Anesthesia, 11, 536-539.

Day, P. (2004). What is the evidence on the safety and effectiveness of the reuse of medical devices labelled as single-use only? New Zeland Health Technology Assessment, Technical Brief Series, 3.

Dunnigan, A.; Roberts, C.; McNamara, M.; Benson, DW. \& Benditt, DG. (1987). Success of reuse of cardiac electrode catheters. American eburnal of Cardiology, 60, 807-810.

EUCOMED. (2002). Patients in danger: the reuse of single use medical devices in Europe. June 2002.

Fedel, M.; Tessarolo, F.; Ferrari, P.; Lösche, C.; Ghassemieh, N.; Guarrera, GM. \& Nollo G. (2006). Functional Properties and Performance of New and Reprocessed Coronary Angioplasty Balloon Catheters. eburnalof Biomedical Materiasl Research B Applied Biomaterials, 78, 364-372.

Fichet, G.; Comoy, E.; Duval, C.; Antloga, K.; Dehen, C.; Charbonnier, A. et al. (2004). Novel methods for disinfection of prion-contaminated medical devices. Lancet, 364, 521526.

GAO. (2000). US General Accounting Office. GAO Report: single-use medical devices: little available evidence of harm from reuse, but oversight warranted. June 20, 2000.

GAO. (2008). US General Accounting Office. GAO Report: Reprocessed single-use medical devices. FDA oversight has increased, and available information does not indicate that use presents an elevated health risk. January 31, 2008.

Granados, DL.; Jimenez, A. \& Cuadrado, TR. (2001). Assessment of parameters associated to the risk of PVC catheter reuse. eburnal of Biomedical Materials Research, 58, 505-510.

Hall, W. (1991). Ethical issues in the reuse of single-use medical products. In "Reuse of singleuse medical devices: reviewing the issues". Chatswood, NSW: Medical Industry Association of Australia Inc., pp. 45-49.

Hambrick, D. (2001). Reprocessing of single-use endoscopic biopsy forceps and snares. One hospital's study. Gastroenterology Nursing, 24, 112-115.

Horowitz, LN. (1986). Safety of electro physiology studies. Circulation, 73, 28-31.

Kinney, TP.; Kozarek, RA.; Raltz, S. \& Attia, F. (2002). Contamination of single-use biopsy forceps: a prospective in vitro analysis. Gastrointestinal Endoscopy, 56, 209-212.

Kozarek, RA.; Raltz, SL.; Ball, TJ.; Patterson, DJ. \& Brandabur, JJ. (1999). Reuse of disposable sphincterotomes for diagnostic and therapeutic ERCP: a one-year prospective study. Gastrointestinal Endoscopy, 49, 39-42.

Lindsay, BD.; Kutalek, SP.; Cannom, DS.; Hammill, SC. \& Naccarelli, GV. (2001). NASPE policy statement. Reprocessing of electrophysiology catheters: clinical studies, regulations, and recommendations. A report of the NASPE Task Force on Reprocessing of Electrophysiological Catheters. Pacing \& Clinical Electrophysiology, $24,1297-1305$. 
Lipp, MD.; Jaehnichen, G.; Golecki, N.; Fecht, G.; Reichl, R. \& Heeg, P. (2000). Microbiological, microstructure, and material science examinations of reprocessed combitubes after multiple reuse. Anesthesia \& Analgesia, 91, 693-697.

Luijt, DS.; Schirm, J.; Savelkoul, PHM. \& Hoekstra, A. (2001). Risk of infection by reprocessed and resterilized virus-contaminated catheters: an in-vitro study. European Heart eburnal, 22, 378-384.

Ma, N.; Petit, A.; Huk, OL.; Yahia, L. \& Tabrizian, M. (2003). Safety issue of re-sterilization of polyurethane electrophysiology catheters: a cytotoxicity study. eburnal of Biomaterials Science, Polymer Edition, 14, 213-226.

Mak, KH.; Eisenberg, MJ.; Plante, S.; Strauss, BH.; Arheart, KL. \& Topol, EJ. (1996). Absence of increased in-hospital complications with reused balloon catheters. American eburnal of Cardiology, 78, 717-719.

NHMRC. (1997). National Health and Medical Research Council. Report of the NHMRC expert panel on re-use of medical devices labelled as single use. Canberra: Commonwealth of Australia, 1997.

O'Donoghue, S. \& Platia, EV. (1988). Reuse of pacing catheters: a survey of safety and efficacy. Pacing \& Clinical Electrophysiology, 11, 1279-1280.

Plante, S.; Strauss, BH.; Goulet, G.; Watson, RK. \& Chisholm RJ. (1994). Reuse of balloon catheters for coronary angioplasty: a potential cost-saving strategy? cburnal of the American College of Cardiology, 24, 1475-1481.

Polisena, J.; Hailey, D.; Moulton, K; Noorani HZ.; Jacobs, P.; Ries, N. et al. (2008). Reprocessing and reuse of single-use medical devices: a national survey of Canadian acute-care hospitals. Infection Control and Hospital Epidemiology, 29, 437439 .

Quian, Z. \& Castaneda WR. (2002). Can labelled single use devices be reused? An old question in the new era. eburnal of the Vascular Interventional Radiology, 13, 11831186 .

Roth, K. Heeg, P. \& Reichl, R. (2002). Specific hygiene issues relating to reprocessing and reuse of single-use devices for laparoscopic surgery. Surgical Endoscopy, 16, 10911097.

Rozenman, Y. \& Gotsman, MS. (1995). Reuse of balloon catheters for coronary angioplasty eburnal of the American College of Cardiology, 26, 840-841.

Ruffy, R. (1995). Catheter ablation in a less privileged country: the importance of spreading the goods. Pacing \& Clinical Electrophysiology, 18, 1463-1464

Rogez-Kreuz, C.; Yousfi, R.; Soufflet, C.; Quadrio, I.; Yan, ZX.; Huyot, V. et al. (2009). Inactivation of animal and human prions by hydrogen peroxide gas plasma sterilization. Infection Control and Hospital Epidemiology, 30, 769-777.

Shaw, JP.; Eisenberg, MJ.; Azoulay, A. \& Nguyen N. (1999). Reuse of catheters for percutaneous transluminal coronary angioplasty: effects on procedure time and clinical outcomes. Catheterization \&Cardiovascular Interventions, 48, 54-60.

Sloan, TW. (2007). Safety-cost trade-offs in medical device reuse: a Markov decision process model. Health Care Manage Science, 10:81-93.

Tessarolo, F.; Fedel, M.; Ferrari, P.; Caola, I.; Guarrera, GM.; Favaretti, C.; Migliaresi, C. \& Nollo, G. (2004a). Reuse of single use devices for interventional cardiology: a HCTA approach. IFMBE Proceedings, 6. 
Tessarolo, F.; Ferrari, P.; Nollo, G.; Motta, A.; Migliaresi, C.; Zennaro, L. et al. (2004b). Evaluation and quantification of reprocessing modification in single use devices in interventional cardiology. Applied Surface Science, 238, 341-346.

Tessarolo, F.; Caola, I.; Fedel, M.; Caciagli, P.; Guarrera, GM.; Nollo, G. (2004c). Effects of chlorine-releasing compounds on medical devices decontamination. eburnal of Applied Biomaterials and Biomechanics, 2, 219.

Tessarolo, F.; Ferrari, P.; Antolini, R. \& Nollo, G. (2005). Radiofrequency ablation on heartequivalent phantom. Functionality testing of percutaneous single-use catheter. In: Recent Advances in Multidisciplinary Applied Physics, Mendez-Vilas A. ed., Elsevier; pp. 229-234.

Tessarolo, F.; Fedel, M.; Nollo, G. \& Motta, A. (2006a). A novel phantom for the in-vitro quantification of electrophysiology catheter slipperiness: implementation and testing. eburnal of Applied Biomaterials and Biomechanics, 4, 70.

Tessarolo, F.; Caola, I.; Nollo, G.; Antolini R.; Guarrera, GM. \& Caciagli, P. (2006b). Efficiency in endotoxins removal by a reprocessing protocol for electrophysiology catheters based on hydrogen peroxide sterilization. eburnal of Hygiene and Environmental Health, 209, 557-565.

Tessarolo, F.; Caola, I.; Caciagli, P.; Guarrera, GM. \& Nollo, G. (2006c). Sterility and microbiological assessment in reusing single-use cardiac electrophysiology catheters. Infection Control and Hospital Epidemiology, 27, 1385-1392.

Tessarolo, F.; Caola, I.; Fedel, M.; Caciagli, P.; Guarrera, GM.; Motta, A. \& Nollo, G. (2007a). Different protocols for decontamination affect cleaning of medical devices. A preliminary electron microscopy analysis. .burnal of Hospital Infection, 65, 326-333.

Tessarolo, F.; Disertori, M.; Caola, I.; Guarrera, GM.; Favaretti, C. \& Nollo, G. (2007b). Health technology assessment on reprocessing single-use catheters for cardiac electrophysiology: results of a three-years study. Conference Proceeding IEEE Engineering in Medicine and Biology Society, 2007, 1758-1761.

Tessarolo, F.; Disertori, M.; Guarrera, GM.; Capri, S. \& Nollo, G. (2009). Reprocessing single use cardiac catheters for interventional cardiology. Potential savings at the laboratory scale and at national level. International burnal of Public Health, 6, 38-47.

Unverdorben, M.; Degenhardt, R.; Erny, D.; Scholz, M.; Wagner, E.; Kohler, et al. (2005). Clinical and angiographic procedural and mid-term outcome with new versus reused balloon catheters in percutaneous coronary interventions. Indian Heart burnal, 57, 114-120.

Vaiktus, P. \& Burlington, VT. (1997). Patients acceptance of reused angioplasty equipment. American Heart eburnal 134, 127-130.

Vezina, DP.; Trepanier, CA.; Lessard, MR.; Gourdeau, M. \& Tremblay C. (2001). Anaesthesia breathing circuits protected by the DAR Barrierbac S breathing filter have a low bacterial contamination rate. Canadian eburnal of Anaesthesia, 48, 748-754.

Zubaid, M.; Thomas, CS.; Salman, H.; Al-Rashdan, I.; Hayat, N.; Habashi, A. et al. (2001). A randomized study of the safety and efficacy of reused angioplasty balloon catheters. Indian Heart eburnal, 53, 167-171.

Krause, G.; Dziekan, G. \& Daschner, FD. (2000). Reuse of coronary angioplasty balloon catheters: yes or no? European Heart eburnal, 21, 185-189. 


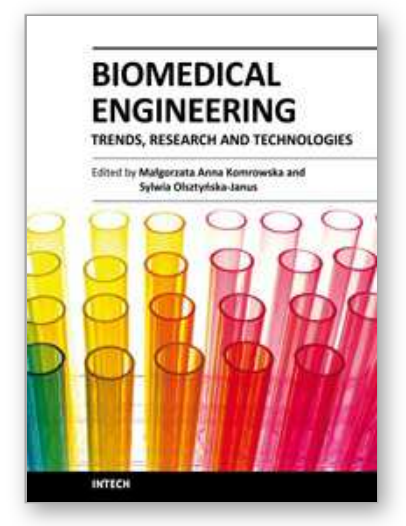

\author{
Biomedical Engineering, Trends, Research and Technologies \\ Edited by Dr. Sylwia Olsztynska
}

ISBN 978-953-307-514-3

Hard cover, 644 pages

Publisher InTech

Published online 08, January, 2011

Published in print edition January, 2011

This book is addressed to scientists and professionals working in the wide area of biomedical engineering, from biochemistry and pharmacy to medicine and clinical engineering. The panorama of problems presented in this volume may be of special interest for young scientists, looking for innovative technologies and new trends in biomedical engineering.

\title{
How to reference
}

In order to correctly reference this scholarly work, feel free to copy and paste the following:

Francesco Tessarolo, Iole Caola and Giandomenico Nollo (2011). Critical Issues in Reprocessing Single-Use Medical Devices for Interventional Cardiology, Biomedical Engineering, Trends, Research and Technologies, Dr. Sylwia Olsztynska (Ed.), ISBN: 978-953-307-514-3, InTech, Available from:

http://www.intechopen.com/books/biomedical-engineering-trends-research-and-technologies/critical-issues-inreprocessing-single-use-medical-devices-for-interventional-cardiology

\section{INTECH}

open science | open minds

\author{
InTech Europe \\ University Campus STeP Ri \\ Slavka Krautzeka 83/A \\ 51000 Rijeka, Croatia \\ Phone: +385 (51) 770447 \\ Fax: +385 (51) 686166 \\ www.intechopen.com
}

\author{
InTech China \\ Unit 405, Office Block, Hotel Equatorial Shanghai \\ No.65, Yan An Road (West), Shanghai, 200040, China \\ 中国上海市延安西路65号上海国际贵都大饭店办公楼 405 单元 \\ Phone: +86-21-62489820 \\ Fax: $+86-21-62489821$
}


(C) 2011 The Author(s). Licensee IntechOpen. This chapter is distributed under the terms of the Creative Commons Attribution-NonCommercialShareAlike-3.0 License, which permits use, distribution and reproduction for non-commercial purposes, provided the original is properly cited and derivative works building on this content are distributed under the same license. 